\title{
A Nobel Prize for genetic scissors
}

\author{
After years of speculation over who would be recognized for the pioneering work on the gene editing tool CRISPR- \\ Cas9, the Nobel Prize in Chemistry has finally been awarded to Emmanuelle Charpentier and Jennifer Doudna.
}

Fis or the first time, the Nobel Prize has been awarded to two women, Emmanuelle Charpentier of the Max Planck Institute and Jennifer Doudna of the University of California, Berkeley, for their work on the development of a method for genome editing. The ground-breaking technology of CRISPR has made substantial contributions to molecular biology and the medical field with a number of promising therapies already in clinical trials. Indeed, a number of CRISPR-based therapeutics and diagnostic tools are currently being investigated to combat the SARS-CoV-2 virus. CRISPR, which stands for clustered regularly interspaced short palindromic repeats, is an adaptive immune system of prokaryotic cells such as bacteria.

The discovery of CRISPR was achieved by the efforts of a number of research groups working on distinct aspects of prokaryote biology. Studies in the late 1980s and early 1990s identified the presence of unusual repeated DNA structures in the genome of Escherichia coli and in the archaea Haloferax mediterranei. Over the next decade, such genomic repeat structures were also discovered in several other prokaryotes, and CRISPR was coined by Ruud Jansen and colleagues in $2002^{1}$. Nonetheless, more significant to the discovery of the CRISPR-Cas9 system was the evidence that CRISPR-containing prokaryotes also had CRISPR-associated (Cas) genes ${ }^{1}$. These Cas genes encoded for proteins with helicase and nuclease activity, having the ability to unwind and cut DNA. Over the following decade, countless studies aimed at identifying the function of the CRISPR-Cas system led to the discovery of its defensive role against viral infection in prokaryotes ${ }^{2}$. However, it was not until 2012, that the potential of the CRISPR-Cas9 system as a gene editing tool was realized.

Emmanuelle Charpentier identified an RNA molecule (tracrRNA) that recognizes foreign genetic material in the bacterium Streptococcus pyogenes. She later collaborated with Jennifer Doudna and they proposed that tracrRNA and CRISPR RNA could be fused together to form a simpler single-guide RNA molecule. This programmable guide RNA can direct Cas9 to cleave sequence-specific regions of $\mathrm{DNA}^{3}$. Around the same time, another group of researchers, led by Virginijus

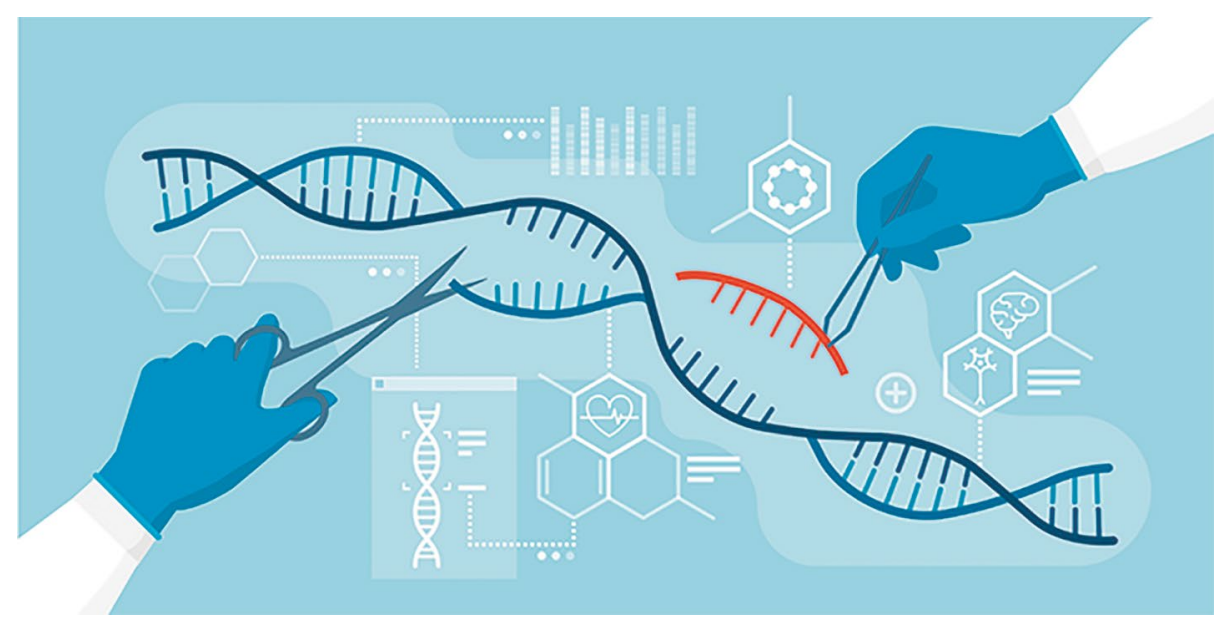

Credit: ElenaBs / Alamy Stock Vector

Šikšnys, also made similar discoveries and were eventually awarded the prestigious Kavli Prize in Nanoscience in $2018^{4}$. Feng Zhang and George Church were the first to demonstrate the potential of CRISPR-Cas9 genome editing in human cells $s^{5}$, which was touted as an even greater achievement due to the complexity of introducing bacterial proteins into human cells. The controversy over the timeline of the discovery has now reached the US Patent and Trademark Office as the respective start-up companies, with the backing of big venture capitalists, scramble to commercialize the multitude of applications for the technology.

The disputes over the invention should not detract from the mammoth contribution that it has made. Compared to other technologies such as zinc-finger nucleases, CRISPR is the simplest and most versatile method for editing the genome sequence in living organisms to date. It is for this reason that it is now widely employed for fundamental research such as in the generation of unique animal models as well as for development of therapies including ex vivo gene-edited cells targeting a range of complications. In one notable example, a patient with HIV was treated with hematopoietic stem cells that were edited with CRISPR-Cas9 to ablate a key co-receptor for HIV infection, CCR5, effectively engineering immunity to HIV infection ${ }^{6}$. There have also been some important milestones in the use of CRISPR in cancer immunotherapy in the generation of chimeric antigen receptor (CAR) T cell therapy. There is also early promise in the use of CRISPR in the treatment of sickle cell disease. CRISPR has also been employed in agriculture to increase crop yield and to generate more disease-resistant plants. It is imperative, however, that good scientific oversight be at the core of research studies using CRISPR in order to avoid unethical experiments, such as the recently purported genetically edited embryos ${ }^{7}$.

The time was certainly ripe for a Nobel Prize for this technology. Emmanuelle Charpentier and Jennifer Doudna as well as other groups are already exploring ways to improve the targeting accuracy of CRISPR. It is clear, however, that the discovery of these genetic scissors has been monumental and the contributions to science and society in general will continue to be realized for a long time to come.

Published online: 18 December 2020 https://doi.org/10.1038/s41563-020-00895-Z

\footnotetext{
References

1. Jansen, R., van Embden, J. D. A., Gaastra, W. \& Schouls, L. M. Mol. Microbiol. 43, 1565-1575 (2002).

2. Barrangou, R. et al. Science 315, 1709-1712 (2007).

3. Jinek, M. et al. Science 337, 816-821 (2012).

4. Gasiunas, G., Barrangou, R., Horvath, P. \& Siksnys, V. Proc. Natl Acad. Sci. USA 109, E2579-E2586 (2012).

5. Cong, L. et al. Science 339, 819-823 (2013).

6. Xu, L. et al. N. Engl. J. Med. 381, 1240-1247 (2019).

7. Cyranoski, D. First CRISPR babies: six questions that remain. Nature (30 November 2018); https://doi.org/10.1038/ d41586-018-07607-3
} 